CAHIERS DE

NARRATOLOGIE

\section{Cahiers de Narratologie}

Analyse et théorie narratives

23 | 2012

Le sujet et l'art dans la prose française contemporaine (1990-2012)

\title{
L'art de faire parler les pierres: Bande-son de Bertrand de la Peine
}

\section{Isabelle Dangy}

\section{(2) OpenEdition}

Journals

Édition électronique

URL : http://journals.openedition.org/narratologie/6619

DOI : 10.4000/narratologie.6619

ISSN : 1765-307X

Éditeur

LIRCES

Référence électronique

Isabelle Dangy, "L'art de faire parler les pierres: Bande-son de Bertrand de la Peine », Cahiers de Narratologie [En ligne], 23 | 2012, mis en ligne le 29 décembre 2012, consulté le 01 mai 2019. URL

http://journals.openedition.org/narratologie/6619; DOI : 10.4000/narratologie.6619

Ce document a été généré automatiquement le 1 mai 2019.

Article L.111-1 du Code de la propriété intellectuelle. 


\title{
L'art de faire parler les pierres: Bande-son de Bertrand de la Peine
}

\author{
Isabelle Dangy
}

1 Pas plus que celles du savant, du médecin ou du criminel, la figure de l'artiste, si privilégiée dans le roman du XIX ${ }^{\mathrm{e}}$ siècle, n'a déserté les récits contemporains où elle épouse la diversification des disciplines artistiques et leur tendance à l'empiétement générique. C'est ainsi que l'on rencontre chez Toussaint, Fleischer ou Houellebecq des plasticiens, des vidéastes et des photographes, mais aussi, plus classiquement, des musiciens et des peintres chez Echenoz, Gailly ou Michon - sans pour autant que les récits concernés se donnent nécessairement pour fin une réflexion sur la nature de l'activité artistique.

2 Celle-ci est au contraire explicitement visée dans Bande-son ${ }^{1}$, deuxième roman de Bertrand de la Peine, paru en 2011 aux éditions de Minuit. La presse littéraire a noté à propos de ce roman une certaine parenté avec l'œuvre de Jean Echenoz ${ }^{2}$, et on verra plus loin qu'il n'est pas étranger à certains traits par lesquels il serait possible de définir une littérature postmoderne, selon les critères de Marc Gontard ${ }^{3}$, puisqu'il présente une discontinuité (relative), pratique la métatextualité et surtout s'inscrit dans le climat de renarrativisation propre au roman contemporain. Peut-on voir dans les tribulations des protagonistes l'émergence d'un Art poétique postmoderne?

3 Si les personnages liés au monde de l'art sont assez nombreux au sein de ce récit qui tourne autour de l'ouverture d'un «Centre des arts du son» dans une ancienne abbaye provençale réhabilitée pour l'occasion, deux d'entre eux se détachent à la fois par leur singularité et la place qu'ils occupent : il s'agit de Sven Langhens, un acousticien danois, et de la figure référentielle de Rudolf Erich Raspe, géologue minéralogiste du XVIII ${ }^{\mathrm{e}}$ siècle plus connu pour avoir écrit les Aventures du baron de Münchhausen ${ }^{4}$. La position semi ironique et apparemment désinvolte du narrateur vis-à-vis de ces deux personnages, de leur caractère, de leurs travaux, de leurs entourages respectifs, et également vis-à-vis de son propre récit, n'exclut pas la quête d'une certaine poésie teintée d'humour : derrière la fonction technique de la régie narrative se dissimule une troisième figure de l'artiste, plus floue et plus indirectement perceptible. On tentera ici d'analyser la manière dont ces trois 
instances (le personnage fictionnel, le personnage référentiel et le narrateur) interagissent pour suggérer le rapport du sujet à la création artistique.

\section{Sven Langhens}

4 Le personnage se définit par sa trajectoire, et par la traversée d'une crise qui éclate vers le tiers du roman avant de se résorber au fil des pages.

$5 \mathrm{Au}$ premier abord l'auteur paraît avoir assemblé autour de Sven Langhens un certain nombre de lieux communs : c'est un homme solitaire, qui vit reclus dans une ancienne magnanerie du Luberon, et se comporte avec une certaine maladresse quand il sort de son antre. Son portrait physique reflète sa vie d'anachorète baba cool : « ses longs cheveux filasse flottent autour des oreilles, sur les lourdes épaules nues, tannées comme celles d'un vigneron " $(B S, 7)$. Ses relations avec sa femme et avec les femmes en général sont empreintes de brusquerie voire d'indélicatesse. Son comportement social est marqué par la timidité et le manque d'aisance, mais sa gaucherie même parait lui réussir en lui attirant la sympathie de ceux qu'il côtoie. Toute cette aura de maladresse ne l'empêche nullement d'entretenir avec des individus très lancés dans les milieux à la mode, comme le couturier et sponsor Franck Castans, les relations nécessaires pour que son œuvre obtienne reconnaissance et légitimité.

6 Mais plusieurs composantes du personnage lui confèrent un statut plus original. Tout d'abord son histoire comporte une assez étonnante reconversion : Langhens est passé de l'image au son, de la peinture à l'acoustique. Le second chapitre du récit récapitule son parcours. Il est venu de Copenhague à Arles sur les traces de Van Gogh, dans le désir de capter quelque chose de la véhémence hallucinée du maître, mais sans grand succès :

Dans les premiers temps, Sven s'installait, dès le lever du jour, sur la terrasse de leur chambre et croquait les tombes voisines, ocrées par l'ombre des platanes. Puis il s'essaya à une étude des Arènes qu'il décida de faire flamber sous le vermillon et le jaune de Naples. Mais son travail acharné n'aboutit qu'à des embus et des tons terreux. Le feu n'avait pas pris. Il persévéra en allant peindre aux abords de la ville. Là encore les cyprès finirent en torchères cendreuses sous un ciel de sang. Son tempérament tenace lui fit cependant poursuivre ses tentatives : coucher sur la toile cette Camargue incandescente. Alors, sous les coups de brosse et de couteau rageurs, les flamants roses devinrent des perroquets, les tamaris, des bougainvilliers et les taureaux, des buffles paissant dans des marécages scintillants de fuchsia et d'indigo. (BS, 17)

7 Ce paragraphe caricature avec férocité les efforts inutiles d'un barbouilleur : c'est donc ici, au seuil du récit, la figure de l'artiste raté qui est convoquée. Cependant l'opiniâtreté $\mathrm{du}$ peintre, ainsi que le choix d'une palette radicale, suggèrent la présence en lui d'une énergie volontaire, d'une réelle vocation créatrice que souligne la récurrence du thème du feu. La suite du récit laisse entendre que Langhens s'était simplement trompé de mode d'expression: son domaine n'est en définitive pas la couleur, mais le son. Et surtout: l'attitude qui lui réussit artistiquement n'est pas celle de la flamboyance extravertie, mais celle d'une écoute patiente, d'une captation de l'infime associée à une manipulation de l'infra auditif. Cela suppose un long apprentissage. Sven réalise donc des enregistrements, les amplifie, les trafique de manière à rendre audible ce qui habituellement échappe à l'oreille humaine : le tapotement des pas des lézards sur la pierre, le bruit d'une épeire dévorant une mouche ou d'un escargot mangeant une feuille de salade. La quête du son, qu'il cherche à agrandir comme on ferait d'une photographie, remplace pour lui non 
seulement la peinture, mais la présence de sa femme, qui a choisi de ne pas prolonger la vie commune. Vincent Roy, dans l'article du Monde où il rendait compte du roman nouvellement paru de Bertrand de la Peine, notait non sans justesse: «La véritable héroïne du roman, c'est la perception ${ }^{5} »$.

8 Autre changement : alors qu'autrefois il n'avait besoin que d'une toile, de pinceaux et de tubes de peinture, la démarche de Langhens le situe maintenant à la frontière de l'art et de la technique, dans cette zone où la création s'effectue moyennant un appareillage sophistiqué («Fostex MR8 MKII» ou «casque molletonné Sennheiser HD25») et une installation minutieusement programmée, que le début du roman décrit au lecteur non sans un zeste d'amusement complice, d'autant que ces coûteuses machines sont mobilisées pour enregistrer une scène aussi triviale que le repas d'un escargot sur une salade. Cependant cet appel à la technologie de pointe et cette concession à la modernité coexistent avec un don de soi total et avec une sorte d'union mystique à la nature. Langhens s'enferme dans la solitude et l'austérité, éduque son corps de manière à lui donner une hypersensibilité auditive, il active en lui pour cela les corpuscules de Pacini " pour mieux appréhender le milieu naturel telle une taupe qui tâtonne dans le dédale d'une galerie » (BS, 26), autrement dit il se fond autant que possible dans le milieu qu'il espère saisir, et la comparaison avec la taupe active un fantasme d'enfouissement récurrent chez ce personnage. Cette aspiration se traduit encore dans le passage suivant :

Grâce au nouveau matériel qu'il pouvait désormais s'offrir, il donna à ses enregistrements une dimension tellurique, en prise directe avec les forces terrestres. Il s'en dégageait une impression abstraite et brute. Quelque chose de radical et de chtonien. On était passé aux choses sérieuses. $(B S, 25)$

Comme on le remarque dans cette dernière phrase, où il est difficile de décider si l'allusion aux choses sérieuses est ou non teintée d'ironie, le personnage de Langhens évolue toujours à la limite du sublime et du ridicule. Son ambition mégalomane se révèle plus encore dans la phase suivante de son travail, quand, après avoir enregistré l'écho minime de certains phénomènes animaux ou végétaux, il s'attaque au monde minéral et décide de faire parler la pierre, ce qui se traduit concrètement par une tentative d'enregistrement en plein midi, dans des carrières proches de chez lui : « Il attend que la roche craque, brûlant sous le soleil, il espère saisir le son des particules de calcaire mêlées au quartz qui, en se disloquant, provoqueront d'ultimes déflagrations» (BS, 30). La tentative échoue, en raison de l'arrivée intempestive d'un escadron d'ULM dont le vacarme vient parasiter le silence nécessaire à l'enregistrement, incident que le narrateur évoque non sans un humour qui se déploie aux dépens de son personnage. La figure de l'artiste, soumise dans le roman à des mésaventures répétées, oscille ainsi entre deux postulations: d'une part elle subit la tentation d'une certaine radicalité monastique, spirituelle, attachée à une descente vertigineuse vers l'infinitésimal, à un retour aux origines, à l'écoute du langage primitif de la pierre, d'autre part elle est malmenée par les contingences du monde moderne qui se jouent de sa vocation, la ridiculisent et l'apparentent à la figure baudelairienne de l'Albatros. De même, la virtuosité technique s'allie chez lui à une profonde humilité, puisque son rôle artistique consiste essentiellement en un captage : le sujet de l'art, s'il entend dépasser la condition humaine en allant vers l'infiniment petit, s'efface devant son objet et se contente d'affiner son aptitude à percevoir. Ensuite, son intervention artistique consistera surtout à amplifier les microsons qu'il aura emprisonnés dans ses appareils, à les rendre audibles pour d'autres. Car Langhens est animé par une volonté naïve de partage et de communication qui le pousse par exemple à présenter ses enregistrements de lézards à la fête du village, 
devant « un parterre de notables, d'agriculteurs et de commerçants chevalblanais » (BS, 24). Sans doute s'accorde-t-il le droit à une manipulation, à un montage des sons enregistrés, à une démarche de transformation qui introduit une dimension musicale dans le colportage sonore, mais le texte tend à minimiser cet aspect du processus artistique. L'artiste demeure désormais à l'arrière-plan de l'œuvre qu'il élabore.

Une autre particularité de Langhens réside dans sa propension aux basculements soudains. Il a besoin, pour exister en tant que sujet artistique, de se déprendre d'un héritage. Petit-fils d'un auteur de marines danois de seconde zone, il a choisi la peinture un peu de manière atavique, même si son inspiration, dans le sillage de Van Gogh, se veut plus méridionale et plus ardente que n'était celle de son aïeul. Il lui faut oublier deux fois sa formation et ses antécédents pour se trouver : oublier Copenhague et son grand-père pour rencontrer le midi, puis oublier les pinceaux et Van Gogh pour rencontrer le son.

11 Il faut ajouter que sa véritable vocation l'atteint à la manière d'une révélation. À première lecture, l'épisode peut sembler anodin : le jour de son arrivée en Provence, Langhens en sortant de sa voiture est assailli et émerveillé par le vacarme des cigales dont le grésillement l'enveloppe comme un bain. Et c'est désormais ce vécu d'immersion que, audelà de la surprise un peu pataude du nordique en pays méditerranéen, il va rechercher en s'enfonçant dans le son ou dans le silence, vécu que l'on peut évidemment rapprocher de son goût pour les grottes et autres lieux chtoniens, et qui correspond à un désir un peu régressif d'enfouissement. Ironiquement, une malédiction relative aux bains semble cependant poursuivre ce personnage avide de s'immerger. Le plaisir de se plonger dans l'eau lui est plusieurs fois refusé, soit en raison de l'état déficient de la baignoire qui lui est attribuée, soit parce que l'occasion ne lui permet pas de nager dans les flots tentants d'une rivière, et ainsi de suite jusqu'à ce qu'il retrouve la solution de l'immersion sonore. Quoi qu'il en soit, en tant que sujet de l'action, il fonctionne par renversements brusques, autrement dit demeure opaque à lui-même jusqu'à ce qu'une prise de conscience lui révèle sa propre vérité. Ce phénomène se reproduit plus tard dans le récit, par exemple lorsqu'il reconnaît d'un seul coup un objet qu'il a regardé jusque-là sans le voir, « un disque minéral de couleur noire » (BS, 85), et l'identifie comme un miroir d'obsidienne dont il tirera des vibrations insoupçonnées. Dans la création artistique interviennent donc la surprise, le basculement.

12 Au-delà du stéréotype initial, le personnage de Langhens propose ainsi une vision de l'artiste assez moderne : capteur plus que créateur, technicien tout autant qu'artisan, il est avant tout issu d'une translation qui l'a fait passer d'un genre traditionnel, la peinture, à un genre innovant, la «bioacoustique », en lui permettant de conquérir dans l'abnégation et l'auto-effacement son identité d'artiste. Cette translation l'a mené de la brûlure vangoghienne au froid de la pierre, de l'extraversion forcenée à une introversion obstinée, de l'expressionnisme au minimalisme. Demeure le mystère de son aspiration à percevoir le chant de la pierre, aspiration qu'il partage, au-delà du décalage chronologique, avec Raspe.

\section{Rudolf Eric Raspe}

13 Le deuxième personnage chargé de représenter le rapport de l'artiste à son art est Rudolf Erich Raspe. Il s'agit d'une figure historique réelle, que l'écrivain traite comme telle en évoquant tel ou tel épisode attesté de son existence, ou encore en mentionnant ses relations avec certains de ses contemporains, l'ingénieur Watt ou l'homme de lettres 
Horace Walpole, mais qu'il manipule à son gré en introduisant des péripéties non authentifiées et en citant des pages de carnets intimes tout aussi apocryphes.

14 Le lien avec Sven Langhens est établi au moyen d'un artifice romanesque plus que répertorié : le danois découvre dans une vieille malle des objets de famille dont un essai de Raspe sur les « pierres chantantes » et, au prix d'une incursion sur Internet, s'aperçoit que ce Raspe n'était autre que l'époux de l'une de ses aïeules. La lecture de l'essai aiguise la curiosité de Langhens, et il s'élance sur les traces de ce nouveau maître (et ancêtre) qui relaie sans le vouloir le magnétisme initiatique du grand-père aux marines et de Van Gogh.

$15 \mathrm{Si}$, avec l'entrée en scène de Sven Langhens, c'était la figure de l'artiste raté qui s'introduisait dans le récit, c'est celle du touche-à-tout qui correspond à l'apparition de Raspe. En effet, bien qu'il se définisse lui-même comme un artiste et demeure navré que son talent soit méconnu (sans que l'on sache très bien laquelle de ses multiples activités mérite le nom d'art, mais sans doute s'agit-il de son travail d'écrivain), Raspe est d'abord l'un de ces savants du XVIII ${ }^{e}$ siècle qui s'intéressèrent à toutes sortes de disciplines, et pour lesquels la frontière entre art, science et technique ne semble pas avoir été une catégorie de pensée très pertinente. Si sa formation première est celle d'un géologue, « les domaines dans lesquels il laissa des écrits vont des volcans aux fossiles, en passant par la mythologie celte et le théâtre médiéval » (BS, 41). D'autres informations survenant plus loin dans le récit indiquent qu'il serait aussi l'auteur non seulement d'une version des célèbres Aventures du baron de Münchhausen mais aussi d'un roman gothique, Hermin et Gunnilde, dans le style du Château d'Otrante, cependant il ne semble pas qu'il faille prendre au sérieux cette attribution. À cela s'ajoute une activité de collectionneur, voire de trafiquant ${ }^{6}$, qui associe son image à celle des cabinets de curiosité de l'Ancien Régime, assemblages d'objets hétéroclites recueillis pour leur beauté, leur rareté ou leur extravagance, à la limite encore une fois du souci scientifique et du souci esthétique. L'inventaire établi par ses soins pour la collection d'objets d'un certain Lord Henry Albert donne une idée de cette fantaisie: on ne peut le citer intégralement, mais on notera le voisinage saugrenu et charmant de spécimens dont le rapprochement évoque l'art du collage, tels que «Une pierre-paysage présentant le dessin d'un château fort (Zlin, Moravie) », "Un nautile pétrifié monté sur un piédouche argenté (mer de Barentz)», « Une griffe de l'oiseau-Roc capable de soulever un bœuf (origine incertaine) » (BS, 78).

16 Certes, les intérêts dispersés de Rudolf Erich Raspe paraissent plus naturels si on les rapporte à son époque, mais ils suggèrent cependant l'image d'un personnage brouillon et d'un artiste quelque peu caricatural, d'autant que les pages de son journal révèlent un individu très terre à terre, préoccupé de ses repas, des visites nocturnes d'une servante, de ses embarras financiers, et péniblement convaincu que le monde entier est responsable de sa disgrâce et refuse de l'admirer à sa juste valeur - oubliant au passage qu'il a lui-même provoqué son exil et la ruine de sa réputation, pour avoir financé ses recherches et ses amours par des «emprunts» dans la collection d'estampes du Landgrave de Hesse, dont il était le conservateur en chef. En somme, la figure de l'artiste ne sort pas ennoblie de cette incarnation haute en couleur, et pourtant, comme dans le cas de Langhens, derrière le personnage picaresque se profile une entité plus subtile et une vision de l'art authentique et crédible. En effet, Raspe est un personnage en quête de quelque chose qu'il ne parvient pas à nommer, qu'il formule vaguement en termes de «mystères de la Nature»(BS, 92), dont la poursuite lui impose cette exigence: «Continuer, à l'écart de mes semblables, seul» (BS, 92). Et son carnet le montre à 
plusieurs reprises en proie, devant certains spectacles, à une extase proche de la révélation :

J'entrepris donc de gravir un tertre non loin de là. De là-haut, la vue est stupéfiante. Le paysage m'est alors apparu, dans son austérité de bruyères et de roc, tel un miroir poli par une formidable volonté démiurgique, miroir où se réfléchissait mon être tout entier. Je fus traversé d'un grand trouble et d'une clairvoyance plus grande encore qui m'obligea à faire halte sur les degrés de pierre. (BS, 100)

Assimiler ainsi le paysage à un miroir est le fait d'une créature qui se cherche, qui, sous le masque de ses identités pittoresques et changeantes, s'interroge sur la nature du beau et sur le sens de sa propre quête. D'autre part le thème du miroir reviendra, on le verra, à la fin du roman. De même, lorsqu'il tombe sur l'énigme naturelle des pierres chantantes, en Westphalie d'abord et ensuite en Irlande, il effectue leur description avec un certain lyrisme, faisant de cette expérience l'occasion d'une réflexion sur son moi. Dans les deux passages suivants on appréciera notamment la recherche stylistique un peu ampoulée avec laquelle l'auteur le fait s'exprimer, et le sens de la métaphore qu'il lui prête :

Sur plusieurs lieues, je parcourus le littoral, envoûté par le spectacle de l'inlassable travail de la mer abrasant la côte rocheuse. Heureux témoin des noces morganatiques de l'émeraude éclatante des prairies avec le noir sec, anguleux, bossué du granit, je fus pris d'une joie toute géologique. (BS, 98)

Moi-même, n'étais-je pas devenu, année après année, couche après couche, un monolithe que les sédiments avaient façonné à mon insu? Le processus de cristallisation ne m'avait-il pas peu à peu gagné au fil du temps, faisant de moi un véritable fossile? Mais quel marteau viendra faire craquer la gangue qui m'enserre, révélant au grand jour mes joyaux enfouis, ma véritable nature d'artiste, mon talent? (BS, 99)

Comme Langhens, Raspe est à la recherche de la forme qui l'exprimera vraiment et le révélera: la conquête de la voie artistique, dans ce roman, n'est pas immédiate, elle suppose errance, erreur et persévérance. Les figures qui l'incarnent sont donc soumises à une sorte de parcours initiatique, et même, si l'on prend au pied de la lettre l'image de la gangue citée un peu plus haut, elles seraient en attente d'une libération, éventuellement brutale, qui permettrait à leur moi pétrifié d'éclater et de libérer une parole.

\section{Le chant de la pierre}

19 Le récit tisse entre les deux artistes un lien qui agit à plusieurs niveaux. Raspe est en premier lieu un stimulateur de l'action: c'est la découverte de son manuscrit qui relance la combativité de Langhens dans un moment de dépression, recrée en lui une excitation et l'élan d'une quête qui va le conduire en Irlande et l'aimanter vers le dénouement du récit. La malle, les documents secrets dormant dans l'oubli, l'Irlande, tout cela tire du côté du conte ou de la ballade un roman qui par d'autres aspects s'inscrit pourtant dans la réalité prosaïque du monde contemporain. Les pierres chantantes sont elles mêmes des objets inclassables, des curiosités acoustiques que Raspe présente comme des raretés naturelles mais que l'on situerait plutôt à mi-chemin entre poésie, magie et observation scientifique. Un climat proche du merveilleux enveloppe certains passages du roman, tandis que la composition centrée sur la quête d'un objet pourrait aisément être analysée selon les fonctions proposées pour le conte par Vladimir Propp ${ }^{7}$.

Langhens prend donc l'avion dans l'espoir de retrouver ces « Singstein » que Raspe aurait découvertes dans les grottes d'Inchfallen, quelque part dans le comté de Glenmay. Il met 
ses pas dans les pas de Raspe, visite son tombeau, entre en possession de son journal, et, même s'il porte sur la personnalité de Raspe un jugement assez critique, accomplit ainsi une sorte de pèlerinage auprès de cet ancêtre avec lequel il se reconnaît au moins une passion commune : le langage des pierres. Les deux artistes ont encore en partage d'être sensibles l'un et l'autre à un sentiment cosmique qui les pousse à se confondre avec la matière et à rechercher le contact de la roche. De plus ils affirment tous deux un goût prononcé pour la technique, chacun à l'échelle de son siècle : Langhens utilise, on l'a vu, des appareils d'enregistrement sophistiqués, tandis que Raspe vend ses compétences en Irlande pour équiper certaines mines de cuivre de machines à vapeur. On voit ici que la figure de l'artiste se dédouble, que le thème se creuse en abyme, ou, si l'on veut, que Raspe est injecté dans le récit de manière spéculaire comme une version plus caricaturale de l'artiste, plus caricaturale dans la mesure où, à un degré supérieur par rapport à Langhens, elle reflète la faiblesse voire la petitesse humaine et les ridicules afférents. Ce qui ne l'empêche pas d'être le médiateur d'une expérience profonde et exaltante.

Grâce à un miroir d'obsidienne découvert en Irlande chez ses hôtes qui l'ont eux-mêmes hérité de Raspe, miroir dont les bords ciselés sont capables, à condition d'être correctement exposés, d'amplifier les inflexions du vent, Langhens parvient en effet à revisiter le rêve de Raspe tout en donnant substance à son propre fantasme : faire chanter la pierre. Avant son arrivée, l'objet sombre et muet demeurait accroché à un mur. Mais, tel un héros de conte encore une fois, Langhens l'identifie, l'éveille et le transforme en œuvre d'art pour l'inauguration du Centre provençal. Ce substitut ne constitue du reste pas son dernier mot sur la question, car la dernière page du roman laisse entendre que le pèlerinage n'est pas terminé, et que Langhens songe à un voyage en Westphalie, toujours sur les traces de son prédécesseur, à la recherche d'autres pierres chantantes que celui-ci y avait à l'origine découvertes.

22 Le désir de faire chanter la pierre ne reçoit pas de commentaire explicite dans le cadre du roman. Il appartient au lecteur d'y voir une quête de l'archaïque, une plongée dans le minéral qui raccorderait l'artiste aux origines de l'univers, une nostalgie polysémique de la grotte et de ses secrets bâillonnés par le passage des siècles. Ou un intérêt pour la matière qui se prêterait à une divinisation panthéiste des forces de la nature. Ou une métaphore du passé, un effort pour donner une voix aux êtres disparus, comme le suggère Marine Landrot dans Télérama $a^{8}$. Ou encore une volonté d'arracher à l'inertie et au silence tout ce qui dans l'homme demeure mutique, pétrifié, inapte à l'expression: la douleur, l'inconscient, l'altérité, le sentiment du sacré...

Pour percevoir ce langage de la pierre, il faut une oreille exercée, et pour le faire percevoir, un système d'amplification. L'oreille, l'amplificateur: c'est à cela que se ramène le travail de l'artiste, et, dans les deux cas, cela induit une posture en retrait, un effacement du sujet par rapport à l'œuvre en cours, une attitude qui relève plus de l'expérimentation ou de la manipulation que de la création pure et simple.

\section{Et l'écrivain?}

Le narrateur pose d'une manière différente la question du rapport à l'art. Il construit, dans Bande-son, la bande-son de l'écriture. Le choix de ce titre est essentiel. Il suggère en effet un écrivain cinéaste qui aurait renoncé à l'image pour ne conserver que son accompagnement sonore : musique, bruitages, dialogues. Pris au pied de la lettre, ce titre inviterait à écouter le récit en aveugle, en amputant l'univers sensoriel du roman de toute 
notation non auditive. En réalité, le parti pris du son n'est pas aussi radical. Cependant on note, de la part de l'écrivain, une attention constante, une attention d'acousticien en quelque sorte, portée à la prosodie et au rythme de la phrase, et le recours à d'autres procédés littéraires, recherches allitératives et sonores notamment, qui tirent Bande-son vers une écriture poétique. Celle-ci se révèle encore dans le goût des énumérations et des listes, procédé ambigu qui concilie l'abondance et la brièveté, comme on s'en rend compte à la lecture du passage suivant, consacré aux attitudes guerrières d'une jeune fille :

Lorsqu'elle officie dans la cuisine, Angelica éventre les paquets de farine.

Elle décapite les bouteilles de lait.

Explose les nouilles.

Pulvérise le riz.

Trucide les boîtes de conserve.

Mutile les pizzas.

Scalpe les tablettes de chocolat.

Terrifie les surgelés.

Armée d'une pince ou d'un tournevis, la jeune fille fait régner dans la pièce un

avant goût de massacre. (BS, 63)

L'antiphrase contenue dans le prénom d'Angélique (et peut-être la relation intertextuelle avec l'héroïne de l'Arioste) souligne le ton parodique utilisé pour la description de gestes qui ne visent finalement qu'à élaborer tourtes et moussakas, tandis que la succession des images induit ponctuellement une atmosphère de surenchère verbale. Ainsi le texte s'imprègne d'une sorte de comique poétique qui n'est pas sans rappeler Michaux. Ailleurs, c'est encore le goût du pastiche qui se manifeste, notamment dans les pages attribuées à Raspe qui imitent avec un plaisir perceptible la rhétorique et les tournures d'un diariste de l'époque des Lumières. Il n'y a donc aucun renoncement à l'écriture de la part du narrateur, qui en cela n'imite pas totalement l'aspiration au dépouillement revendiquée par son personnage principal.

D'autre part, si les péripéties du roman se traduisent par de nombreuses notations sonores (pétarades, chants d'oiseaux, roulis des valises dans une gare, jurons, portières qui claquent) et intègrent de nombreux passages dialogués souvent masqués par le discours indirect libre, l'écrivain ne s'interdit nullement de faire défiler des images en parallèle avec la série d'indications auditives. Bien au contraire, il met à profit le passé de peintre de son personnage central pour diffuser en nombre de passages une certaine intensité visuelle. Les images du monde apparaissent volontiers comme autant de pans géométriques colorés à la manière d'un tableau abstrait. Ainsi par exemple, lorsque Langhens découvre au cours d'un bref séjour parisien que sa femme a décidé de le quitter, la situation se présente à lui de la manière suivante : « La porte du palier est verrouillée et lorsque Sven la pousse, la clarté solaire qui frappe le petit appartement blanc l'aveugle. Blanc, jaune. Jaune, blanc. Une constellation de carrés jaunes se révèle à présent » (BS, 34). Il s'avère rapidement que les carrés jaunes ont un rôle fonctionnel : ce sont des postit que son épouse a collés sur leurs biens respectifs en vue d'une répartition à l'amiable. Cependant il y a indéniablement dans cette vision un caractère pictural, surtout si l'on se souvient que le couple sur le point de se séparer avoue une prédilection pour Mondrian. Malgré cela, la domination habituelle du visuel sur l'auditif et sur les autres impressions sensorielles est certainement infléchie et modifiée, à l'échelle du roman, dans le texte de Bertrand de la Peine.

Et surtout, comme au cinéma, cette bande-son a subi les aléas d'un montage. La narration joue constamment de l'ellipse et des abrégés, progresse tantôt par fondus enchaînés, 
tantôt par failles abruptes, à tel point qu'on a pu écrire que le roman devait plaire aux " amateurs de free-jazz ${ }^{9}$ ». Le récit au présent associé à l'usage d'un lexique très étendu qui n'exclut pas le recours à des termes familiers ou populaires donne au roman une alacrité qui ne cesse de pousser la lecture vers l'avant. Ce rythme est d'autant plus sensible que le ton employé évite toute lourdeur émotionnelle, favorise la sensation plutôt que la notation psychologique, infiltre dans le texte un apport constant d'ironie indulgente et d'humour.

En effet le narrateur porte sur le monde des artistes un regard distancié, amusé et parfois même satirique. Son approche de la création contemporaine est teintée d'ironie. On a pu constater qu'il s'amuse volontiers des croûtes de Langhens ainsi que de ses manipulations acoustiques tâtonnantes et même de ses déboires. Mais la satire ne s'arrête pas au personnage principal. Autour de lui le narrateur suggère la présence et le bouillonnement d'une coterie que l'on devine à la fois snob et intéressée. Le couturier Franck Castans, qui sponsorise les recherches de Langhens et de quelques autres, le fait au moins en partie dans un but publicitaire : s'il supervise, de manière assez convenue, la réhabilitation de l'abbaye de Montdragon en Provence afin d'en faire un Centre des arts du son, c'est aussi parce que le défilé de sa collection d'hiver doit avoir lieu dans ce décor original un mois après l'inauguration dudit centre. Quant à cette inauguration, elle est décrite sur un ton discrètement persifleur:

Le vent est dehors. Il survole le rectangle du cloître du cimetière où nulle croix, nulle stèle ne se dresse. Les frères étaient ensevelis sans sépulture aucune. C'est précisément l'endroit qu'a choisi Franck Castans pour inaugurer le Centre et une jolie foule piétine la terre consacrée. Les pans des nappes battent contre les pieds des tables et du buffet aménagés dans l'enclos ceint d'arc-boutants de pierre. Les gens de la Drac sont là, dans leur apparente simplicité. Des top-models plaquent des robes arachnéennes sur ce qu'il leur reste de cuisses. D'autres maintiennent de deux doigts les ailes de leurs capelines. Le maire de Saint-Pantaléon a pris la parole pour se féliciter qu'un enfant du pays ait eu l'idée de redonner vie à cette bâtisse depuis trop longtemps livrée aux ronces et aux éboulis. La griffe de notre grand couturier est devenue synonyme de luxe et de prestige, prestige qui rejaillira sur l'abbaye de Montdragon. Le bâtiment remis à neuf pourra offrir aux visiteurs des expositions de qualité; aux amateurs et aux professionnels les colloques! les séminaires! Et bien sûr des résidences d'artistes sensibiliseront la population à la démarche de création... (BS, 120)

Les considérations culturelles du maire cachant mal son intérêt pour les retombées financières de l'opération, les mondanités (tables du buffet et cuisses des top-models) sur l'emplacement même où les moines sont ensevelis dans le plus total dépouillement, tout cela relèverait presque de la charge. Le narrateur ne cherche pas à estomper l'idée que l'art contemporain est affaire d'argent, de relations, de mode également. Cependant cette satire est sans agressivité profonde, comme si les pratiques culturelles telles que les colloques, séminaires et résidences d'artiste qui entourent la création, donnant un cadre officiel au travail de l'artiste, n'empêchaient pas réellement sa liberté de s'exprimer. D'une manière analogue la description des œuvres exposées pour cette inauguration jette le trouble dans l'esprit du lecteur, car elle oscille constamment entre sérieux et burlesque. Par exemple il est question d'une installation de Tristan Demaze intitulée J'étais un acouphène: «Des impulsions de bruits industriels parasitent le lieu [il s'agit du prieuré] durant quelques fractions de seconde, crépitent autour des ogives, hachurent les piliers, zèbrent le jardinet de buis taillé en son milieu » (BS, 118). Si le titre de l'œuvre prête à sourire, son aspect acoustique n'a rien de particulièrement loufoque et rappelle 
effectivement certaines recherches musicales contemporaines. Peut-être cette posture frontalière, qui maintient le narrateur à la limite de l'adhésion, de l'indulgence et de l'ironie, laisse-t-elle entendre que l'art, à l'instar des personnages qui le représentent dans le récit, doit chercher, errer pour trouver éventuellement, et qu'une quête sincère ne peut éviter parfois de frôler l'absurde ou le ridicule. De plus l'installation de Tristan Demaze relève aussi d'un certain minimalisme, par son aspect fragmentaire et convulsif, ainsi que par sa brièveté répétitive. Elle n'est pas sans analogie avec l'effort du narrateur pour dégraisser, aller à l'essentiel. Il est vrai que le minimalisme, regroupant des réalités littéraires trop disparates, peine à être rigoureusement conceptualisé. On l'entend ici dans le sens d'une tension vers la concision, la fulguration, le silence. Et, en définitive, cette notion, plus que le postmodernisme, semble convenir pour exprimer la posture qui est à la fois celle de l'écrivain et celle de Langhens.

La dernière page du roman ramène en tout cas l'art à sa configuration minimale. L'œuvre exposée par Langhens lors de l'inauguration n'est pas même de sa fabrication au sens strict : le miroir d'obsidienne hérité de Raspe, simplement posé sur un socle de marbre, se voit offert aux modulations du vent dans le séchoir jouxtant la buanderie des moines de Montdragon. Il est vrai que des capteurs sonores se chargent d'amplifier son chant, « afin que les sons sortant de la pierre soient diffractés à l'infini» (BS, 122), et le résultat ressemble à une "envoûtante litanie » (BS, 122), mais le rôle joué par l'artiste est extrêmement ténu. On ne saurait imaginer moins de narcissisme autour d'un objet aussi chargé que le miroir : pourtant ce projet artistique minimal rejoint - paradoxe désormais banal $^{10}$ - une ambition maximaliste, dans la mesure où il ne s'agit de rien de moins que de capter à travers la mélopée du vent le chant du monde, quelque chose comme l'harmonie des sphères.

31 La relation du sujet à l'art se révèle en définitive assez complexe, dans la mesure où elle se propose ici comme la résultante de trois postures distinctes qui sont celles $d u$ protagoniste, de son double, et de son narrateur. Du texte émane une vision de l'artiste héritée du romantisme mais revisitée par le minimalisme: l'activité artistique suppose don de soi et abnégation, solitude et persévérance au cours d'un itinéraire initiatique qui passe par des instants fulgurants de révélation. Cependant cette activité ne peut se déployer à l'heure actuelle que dans un cadre technologique sophistiqué et dans les jupes d'un mécénat mondain. Enfin le déroulement du roman évolue vers une vision de l'art de plus en plus dépouillée mais de plus en plus ambitieuse, qui se confond à la dernière page avec le montage d'un dispositif oraculaire d'un nouveau genre, opération où le sujet artiste semble s'abolir derrière un objet insaisissable. De même que la voix des dieux s'exprimait en bruissant dans les branches des chênes de Dodone, de même le visiteur du Centre de Montdragon pourra ouïr à travers le miroir sonore un langage impalpable ayant la consistance du vent. Le miroir mystique accomplit ainsi les noces de la pierre et de l'air, et c'est aux vents que le roman de Bertrand de la Peine emprunte à son tour la puissance du souffle, le sens de la modulation, des accalmies et des tourbillons, un rythme primesautier et la connaissance de l'espace ${ }^{11}$. 


\section{ANNEXES}

Isabelle Dangy est l'auteur d'une thèse portant sur le thème de l'énigme dans l'œuvre de Georges Perec, publiée en 2002 chez Champion. Son travail actuel concerne l'œuvre de divers écrivains contemporains tels que Jean Echenoz, Olivier Rolin, Christian Garcin, Bertrand de la Peine, Jean-Loup Trassard, Jean Philippe Toussaint, Patrick Modiano, Christian Gailly, Anne-Marie Garat, à propos desquels elle a écrit un certain nombre d'articles, mais aussi à celle de romanciers antérieurs tels que Jules Romains ou André Dhôtel. Elle est membre de l'Association Georges Perec et continue à travailler sur l'œuvre de cet auteur. Elle s'intéresse également à la littérature japonaise, et aux échanges qui ont lieu à l'époque contemporaine entre la littérature française et les cultures orientales.

\section{NOTES}

1. Bertrand de la Peine, Bande-son, Paris, Minuit, 2011 (BS pour les notes suivantes).

2. Notamment Marine Landrot, dans un article paru dans Télérama le 5 janvier 2011, «Bertrand de La Peine, Bande-Son »,Télérama, n³182, 5 janvier 2011.

3. Marc Gontard, "Le postmodernisme en France, définition, critères, périodisation ", Limag. Littératures $d u \quad$ Maghreb, URL: http//www.limag.refer.org/Cours/Documents/ GontardPostmod.htm. Consulté le 15 juillet 2012. Article paru dans Le Temps des Lettres. Quelles périodisations pour l'histoire de la littérature française du 20ème siècle? M. Touret et F. Dugast-Portes (dir.), coll. Interférences, PUR, Rennes, 2001, p. 283-294.

4. Les Aventures du baron de Münchhausen furent publiées pour la première fois en anglais à Oxford en 1785. Le livre n'eut aucun succès, si bien que Raspe vendit ses droits à un éditeur qui fit écrire par un anonyme quatorze nouveaux chapitres. Seuls les cinq premiers sont de la main de Raspe. 5. Vincent Roy, « La savante composition d'un désordre », Le Monde, 11 février 2011.

6. La fragile frontière entre archéologue, collectionneur et trafiquant était aussi l'un des thèmes centraux du premier roman de Bertrand de la Peine, Les Hémisphères de Magdebourg, paru en 2009 aux Éditions de Minuit.

7. Vladimir Propp, Morphologie du conte, Paris, Seuil (Poétique), 1965.

8. La journaliste écrit notamment: "À travers sa quête du singstein, Sven Langhens cherche à entendre la voix intérieure des êtres qui l'ont précédé sur terre, dans l'espoir qu'ils lui transmettent le secret de leur vie », Télérama, 5 janvier 2011.

9. Julien Bisson, « Bande (très) originale », Lire, février 2013.

10. Lionel Ruffel, lors d'une communication prononcée à Cerisy en 2003, «Le minimal et le maximal, ou le renouvellement» (Romanciers minimalistes 1979-2003, Presses Sorbonne nouvelle, 2012) envisageait dans une perspective liée à l'histoire les points de contact possibles entre minimalisme et maximalisme. Depuis l'idée a fait fortune.

11. Au point que l'on pourrait ici discerner les linéaments d'une poétique évocatrice de Reverdy ou de Saint-John Perse. 


\title{
RÉSUMÉS
}

Bande-Son, de Bertrand de la Peine, fait défiler trois figures de l'artiste qui suggèrent pour l'activité artistique un domaine de définition. À la pointe de la contemporanéité, Sven Langhens est un acousticien qui s'attache à rendre audible le chant de l'infime, celui des bêtes minuscules et même celui des pierres. Avant lui, au XVIII ${ }^{\mathrm{e}}$ siècle, Rudolf Erich Raspe, auteur des Aventures du baron de Munchhausen, s'était déjà lancé sur les traces de pierres chantantes. Enfin, légèrement en retrait par rapport à ces deux figures originales dont il orchestre les rapports, le narrateur propose une poétique reflétant et court-circuitant à la fois les recherches de ses deux personnages. Leur interaction culminera dans une installation en Provence, qui, transcendant la distinction entre les différents sujets de l'art et leur objet, parviendra à piéger dans un miroir la force du vent.

Bertrand de la Peine Bande-Son scrolls three artist figures who suggest a domain of definition for artistic activity. At the forefront of the contemporary, Sven Langhens is an acoustician who strives to make audible the voice of the tiny, tiny animals and even the stones. Before him, in the eighteenth century, Rudolf Erich Raspe, author of The Surprising Adventures of Baron Munchausen, had already run on the footsteps of singing stones. Finally, slightly down compared to the two original figures, the narrator reports their relationships and offers a poetic reflecting and bypassing his characters research. Their interaction will culminate in an installation in Provence, which transcends the distinction between the different subjects of art and object, succeeding in trapping the wind by a mirror's force.

\section{INDEX}

Index géographique : Europe

Index chronologique : XXIe siècle

Mots-clés : acousticien, infime, infini, installation, milieux de l'art, minimalisme, peinture, pierres chantantes, reconversion, talent

\author{
AUTEUR \\ ISABELLE DANGY \\ Université de de Saint-Étienne (CIEREC)
}

